\title{
Wavefield Seperation by Energy Norm Born Scattering
}

\author{
Bingbing Sun and Tariq Alkhalifah, King Abdullah University of Science and Technology
}

\section{SUMMARY}

In Reflection Based Waveform Inversion, the gradient is computed by cross-correlating the direct and Born scattered wavefield with their adjoints applied to the data residuals. In this case, the transmitted part of the Born scattered wavefield produces high wavenumber artifacts, which would harm the convergence of the inversion process. We propose an efficient Energy Norm Born Scattering (ENBS) to attenuate the transmission components of the Born modeling, and allow it to produce only reflections. ENBS is derived from the adjoint of the Energy Norm (inverse scattering) imaging condition and in order to get deeper insights of how this method works, we show analytically that given an image, in which reflectivity is represented by a Dirac delta function, ENBS attenuates transmission energy perfectly. We use numerical examples to demonstrate that ENBS works in both the time and the frequency domain. We also show that in reflection waveform inversion (RWI) the wave path constructed by ENBS would be cleaner and free of high wavenumber artifacts associated with conventional Born scattering.

\section{INTRODUCTION}

As conventional Full Waveform Inversion (FWI) (Virieux and Operto, 2009) relies on low frequencies in the data and is prone to "cycle-skipping", especially in dealing with reflections, reflection based Waveform Inversion (RWI) provides a stable alternative inversion of the background model (Xu et al., 2012; Irabor and Warner, 2016). The gradient in RWI is computed by cross-correlating the the back propagated residual wavefield with the demigrated one (the born scattered wavefield). As conventional born scattering produces both reflections and transmissions, the constructed gradient will not only contain the low wavenumber wavepath component for macro velocity updating, but also high-wavenumber migration ellipses, that may induce cycle skipped gradients. Thus, one content of the RWI is for effectively removing those high wavenumber components and it is related to separation of reflection and transmission wavefields.

To attenuate such high wavenumber artifacts, we need to remove the transmission component of the Born scattered energy. There are a few methods available to do so, including for example using Hilbert Transform (Liu et al., 2011) to separate up and down going wavefields or splitting the wave equation (Irabor and Warner, 2016). However, such approaches are relatively expensive: for example, the method based on Hilbert Transform needs extra convolution operations in each time step during the imaging process while the wave equation splitting method requires solving the wave equation with more auxiliary variables and both of these methods can only deal with up-going and down-going waves and can not distinguish between reflection and transmission wavs propagating horizontally.

In this abstract, we propose the Energy Norm Born Scattering (ENBS) method. It can eliminate the transmission waves in born modeling effectively and it works for both vertical and horizontal propagated wavefield. In theory, It is the adjoint operator of the Energy Norm (inverse scattering) imaging condition (Whitmore and Crawley, 2012; Rocha et al., 2016). Thus we would at first describe the method by the adjoint analysis of the ENIC and then by high frequency asymptotic analysis, we shows that analytically this method produces pure reflections when the image or the velocity perturbation is given by Dirac delta functions. We illustrate that the method can remove transmission waves effectively in both the time and frequency domains. At last, we use the proposed method to obtain a clean gradient for velocity updating in RWI.

\section{THEORY}

adjoint of the energy norm imaging condition

The Energy Norm Imaging Condition (ENIC) for Reverse Time Migration (RTM) is described in Rocha et al. (2016) as

$$
I=\int \frac{1}{v^{2}} \frac{\partial S}{\partial t} \frac{\partial R}{\partial t}-\nabla S \cdot \nabla R \mathrm{~d} t
$$

where $S$ and $R$ are the source and receiver wavefields respectively, $v$ is the velocity, and $I$ is the image. $\nabla=\left(\frac{\partial}{\partial x}, \frac{\partial}{\partial y}, \frac{\partial}{\partial z}\right)$ is the space gradient operator. It can also be expressed in the frequency domain using Green functions:

$$
\begin{aligned}
I(\mathbf{x}) & =\iint\left[-\frac{\omega^{2}}{v(\mathbf{x})^{2}} \overline{w(\omega) G\left(\mathbf{x}, \mathbf{x}_{s}, \omega\right)} G\left(\mathbf{x}, \mathbf{x}_{r}, \omega\right)\right. \\
& \left.-\nabla \overline{G\left(\mathbf{x}, \mathbf{x}_{s}, \omega\right)} \cdot \nabla G\left(\mathbf{x}, \mathbf{x}_{r}, \omega\right) \overline{w(\omega)}\right] d\left(\mathbf{x}_{r}, \mathbf{x}_{s}, \omega\right) d \mathbf{x}_{r} d \omega
\end{aligned}
$$

where $\mathbf{x}=(x, y, z)$ represent the Cartesian coordinates, $\mathbf{x}_{s}$ and $\mathbf{x}_{r}$ are the source and receiver coordinates, $w(\omega)$ and $d\left(\mathbf{x}_{r}, \mathbf{x}_{s}, \omega\right)$ are the source wavelet and the record data, respectively. Over line denotes the complex conjugate and $G(\mathbf{b}, \mathbf{a}, \omega)$ represents the Green function from position $\mathbf{a}$ to $\mathbf{b}$ with: $G(\mathbf{a}, \mathbf{b}, \omega)=$ $\overline{G(\mathbf{b}, \mathbf{a}, \omega)}$.

ENIC of equation (2) defines the imaging operator $\mathscr{M}: \quad d \rightarrow$ $I$, its adjoint operator would be a form of Born modeling (or demigration), which produces data given an image: $\mathscr{M}^{*}: \quad I \rightarrow$ $d$. Taking the adjoint of operator in equation (2), and apply it 
to image, we obtain

$$
\begin{aligned}
d\left(\mathbf{x}_{r}, \mathbf{x}_{s}, \omega\right) & \\
= & \int\left[-\frac{\omega^{2}}{v(\mathbf{x})^{2}} \overline{w(\omega) G\left(\mathbf{x}, \mathbf{x}_{s}, \omega\right)} G\left(\mathbf{x}, \mathbf{x}_{r}, \omega\right)\right. \\
- & \left.\nabla \overline{G\left(\mathbf{x}, \mathbf{x}_{s}, \omega\right)} \cdot \nabla G\left(\mathbf{x}, \mathbf{x}_{r}, \omega\right) \overline{w(\omega)}\right]^{*} I(\mathbf{x}) d \mathbf{x} \\
= & \int-\frac{\omega^{2}}{v(\mathbf{x})^{2}} w(\omega) G\left(\mathbf{x}, \mathbf{x}_{s}, \omega\right) \overline{G\left(\mathbf{x}, \mathbf{x}_{r}, \omega\right)} I(\mathbf{x}) \\
- & \nabla \cdot\left[\nabla G\left(\mathbf{x}, \mathbf{x}_{s}, \omega\right) I(\mathbf{x})\right] \overline{G\left(\mathbf{x}, \mathbf{x}_{r}, \omega\right)} w(\omega) d \mathbf{x} \\
& =\int-\frac{\omega^{2}}{v(\mathbf{x})^{2}} w(\omega) G\left(\mathbf{x}, \mathbf{x}_{s}, \omega\right) I(\mathbf{x}) G\left(\mathbf{x}_{r}, \mathbf{x}, \omega\right) \\
- & \nabla \cdot\left[\nabla G\left(\mathbf{x}, \mathbf{x}_{s}, \omega\right) I(\mathbf{x})\right] G\left(\mathbf{x}_{r}, \mathbf{x}, \omega\right) w(\omega) d \mathbf{x}
\end{aligned}
$$

where $*$ denotes the adjoint operation, and over line represents the complex conjugate. We re-express equation 3 using a partial differential equation (PDE) in the time domain as:

$$
\frac{1}{v^{2}} \frac{\partial^{2} p}{\partial t^{2}}=\Delta p+\frac{1}{v^{2}} \frac{\partial^{2} u}{\partial t^{2}} \delta v-\nabla \cdot(\delta v \nabla u)
$$

where, we replaced the image $I(\mathbf{x})$ with the velocity perturbation $\delta v$ here, $u$ is the incident (background) wavefield and $p$ is the Born scattered wavefield.

\section{high frequency asymptotic analysis}

In RWI, the velocity perturbation $\delta v$ is a band limited Dirac delta function. In the following, we will demonstrate mathematically that when we consider the velocity perturbation to be a Dirac function, ENBS given by equation (4) will produce pure reflections.

As the background wavefield $u$ satisfies the wave equation

$$
\frac{1}{v^{2}} \frac{\partial^{2} u}{\partial t^{2}}=\nabla \cdot \nabla u
$$

The source term for ENBS of equation (4 ) can be simplified as

$$
f=\frac{1}{v^{2}} \frac{\partial^{2} u}{\partial t^{2}} \delta v-\nabla \cdot(\delta v \nabla u)=-\nabla \delta v \cdot \nabla u
$$

In the frequency domain, the scattering wavefield can also be expressed as a 3D integration using the Green function:

$$
p\left(x_{0}, y_{0}, z_{0}, \omega\right)=\iiint-\nabla \delta v \cdot \nabla u \frac{e^{i \omega R}}{R} \mathrm{~d} V
$$

where $R=\sqrt{\left(x-x_{0}\right)^{2}+\left(y-y_{0}\right)^{2}+\left(z-z_{0}\right)^{2}}$.

Suppose the velocity perturbation is a Dirac delta function $\delta v=$ $\delta(z)$. Equation (7) can be simplified as

$$
p\left(x_{0}, y_{0}, z_{0}, \omega\right)=\iint\left[\frac{\partial}{\partial z}\left(\frac{\partial u}{\partial z} \frac{e^{i \omega R}}{R}\right)\right]_{z=0} \mathrm{~d} x \mathrm{~d} y .
$$

Here we use the shifting properties for the Dirac delta function:

$$
\int \frac{\partial \delta(z)}{\partial z} q(z) \mathrm{d} z=-\left.\frac{\partial q(z)}{\partial z}\right|_{z=0}
$$

Considering the incident wavefield as a plane wave, we have:

$$
\begin{gathered}
u=e^{i\left(k_{x} x+k_{y} y+k_{z} z\right)}, \quad k_{x}^{2}+k_{y}^{2}+k_{z}^{2}=\frac{\omega^{2}}{v^{2}}, \\
\frac{\partial u}{\partial z}=i k_{z} u, \quad \frac{\partial^{2} u}{\partial z^{2}}=-k_{z}^{2} u .
\end{gathered}
$$

The derivative for the Green function can be evaluated as

$$
\begin{aligned}
\frac{\partial}{\partial z}\left(\frac{e^{i \omega R / v}}{R}\right) & =\frac{e^{i \omega R / v}}{R}\left(i \frac{\omega}{v}-\frac{1}{R}\right) \frac{\partial R}{\partial z} \\
& \approx \frac{e^{i \omega R / v}}{R} i \frac{\omega}{v} \frac{\partial R}{\partial z},
\end{aligned}
$$

where for the far field approximation, we assume that $1 / R<<$ $\omega / v$.

Substitution of the derivatives of equation ( 10 ) and ( 11 ) into equation (8), we obtain

$$
p\left(x_{0}, y_{0}, z_{0}, \omega\right)=-\iint\left[u \frac{e^{i \omega R / v}}{R}\left(k_{z}^{2}-k_{z} \frac{\omega}{v} \frac{z_{0}}{R}\right)\right]_{z=0} d x d y .
$$

It is an oscillatory integral and can be approximated using the stationary phase method. The phase of this oscillatory integral is expressed as:

$$
\Phi(x, y)=\frac{R}{v}+\frac{k_{x} x}{\omega}+\frac{k_{y} y}{\omega} .
$$

The stationary phase approximation gives the result in the form of

$$
p=-c\left[u \frac{e^{i \omega R / v}}{R}\left(k_{z}^{2}-k_{z} \frac{\omega}{v} \frac{z_{0}}{R}\right)\right]_{(x, y)=\left(x^{*}, y^{*}\right), z=0},
$$

where $c$ is the weighting term at the stationary point. $\left(x^{*}, y^{*}\right)$ is the stationary point; it is calculated by setting the gradient of the phase of equation (13) to zero, i.e., $\nabla \Phi=0$ :

$$
\begin{aligned}
& \frac{\partial \Phi}{\partial x}=\frac{x^{*}-x_{0}}{R v}+\frac{k_{x}}{\omega}=0 ; \\
& \frac{\partial \Phi}{\partial y}=\frac{y^{*}-y_{0}}{R v}+\frac{k_{y}}{\omega}=0 .
\end{aligned}
$$

Thus, at the stationary point, we have

$$
\begin{aligned}
k_{x}^{2}+k_{y}^{2} & =\frac{\omega^{2}}{v^{2}}\left[\frac{\left(x^{*}-x_{0}\right)^{2}}{R^{2}}+\frac{\left(y^{*}-y_{0}\right)^{2}}{R^{2}}\right] \\
& =\frac{\omega^{2}}{v^{2}}-\frac{\omega^{2}}{v^{2}} \frac{z_{0}^{2}}{R^{2}} .
\end{aligned}
$$

Using the dispersion relation for plane waves in equation (10), it can be simplified as

$$
k_{z}^{2}=\frac{\omega^{2}}{v^{2}} z_{0}^{2} / R^{2} \quad \text { or } \quad R=\frac{\omega}{v\left|k_{z}\right|}\left|z_{0}\right| .
$$

Substitute this equation into equation (14), we obtain the Born wavefield as

$$
p=-c\left[u \frac{e^{i \omega R / v}}{R}\right]_{(x, y)=\left(x^{*}, y^{*}\right), z=0}\left[k_{z}^{2}-\operatorname{sign}\left(k_{z}\right) k_{z}^{2} \frac{z_{0}}{\left|z_{0}\right|}\right] .
$$

Considering the term in the second brace, It is clear that if the incident wavefield is down going with $k_{z}>0$, the Born wavefield using ENBS would be zero for $z_{0}>0$ and it corresponds to the zone for transmission waves in this setup. In the derivations, we assumed the velocity perturbation or the image as a Dirac delta function. As we pointed out before, in RWI the image would actually be a band limited Dirac function, we show that in this slightly degenerated situation, the proposed ENBS can still perform well in attenuation of transmission waves. 


\section{EXAMPLES}

In the first example, we show an application of ENBS in the time domain. In Figure 1a, we place the source in the upperleft side of the model (denoted by the yellow star) and set the velocity perturbation (image) as a band limited Dirac delta function. The main frequency for modeling is $10 \mathrm{~Hz}$ and the background velocity is set to be constant and equal to $2 \mathrm{~km} / \mathrm{s}$. Figures $1 \mathrm{~b}$ and $1 \mathrm{c}$ are snap shots of the Born scattered wavefield at $t=2.8 \mathrm{~s}$ for the conventional Born and for ENBS, respectively. From this result, it is obvious that ENBS can attenuate transmission waves in the scattered wavefield effectively for both vertical and horizontal propagated incident wavefields.

The same features holds in the frequency domain by solving for the scattered wavefield using the Helmholtz wave equation with the proper source function. This time the source is placed on the surface in the middle (yellow star in Figure 2a, and the background velocity is $2 \mathrm{~km} / \mathrm{s}$. Figure 2 a shows the velocity perturbation; it is a two-reflector model, the reflectivity is also given by a band limited Dirac delta function. The real part of the scattered wavevfield for a $10 \mathrm{~Hz}$ frequency for the conventional Born and ENBS are shown in Figures $2 b$ and 2c ,respectively. The results are in line with the theoretical prediction: the transmission wavefield is attenuated effectively in the frequency domain as well as in the time domain.

The last example corresponds to the computation of the wave path update in RWI. Figures $3 \mathrm{a}$ and $3 \mathrm{~b}$ show the gradients computed using conventional born modeling and ENBS, respectively. We can see that the migration ellipse is attenuated successfully in the ENDS implementation.Controlling the wavepath direction of the scattered field to isolate, for example, reflections has many applications beyond RWI. Even least square migration can attain higher resolution with such a feature. We will discuss such these additional features and show examples in the presentation of this work.

\section{CONCLUSIONS}

We proposed an effective and efficient method to attenuate transmissions in Born modeling, which we refer to as the Energy Norm Born Scattering (ENBS). We showed analytically that given a velocity perturbation represented by a Dirac delta function, ENBS produces pure reflections, i.e., transmission energy is attenuated. Numerically, similar observations are realized with band limited Delta functions. Specifically, the numerical examples show that the proposed method produces pure reflections in both the time and frequency domains. Using ENBS, we can produce a smooth gradient in RWI free of high wavenumber artifacts, which is essential for good convergence of the inversion process.

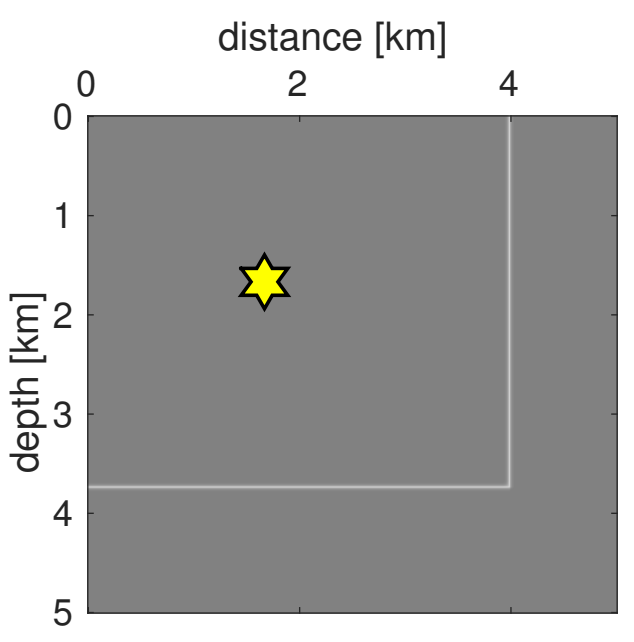

(a)

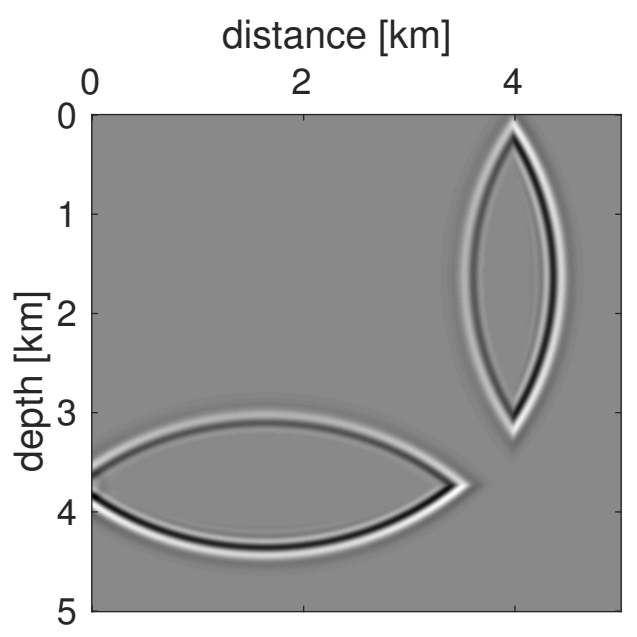

(b)

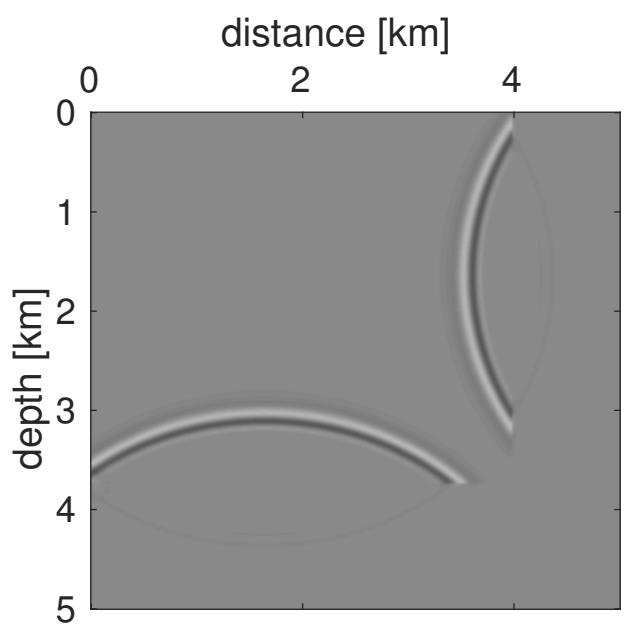

(c)

Figure 1: Application of ENBS in time domain : (a) The velocity perturbation, where the yellow star represents the source position. The scattered wavefiled using (b) the conventional Born modeling, and using (c) ENBS.

Page 1483 

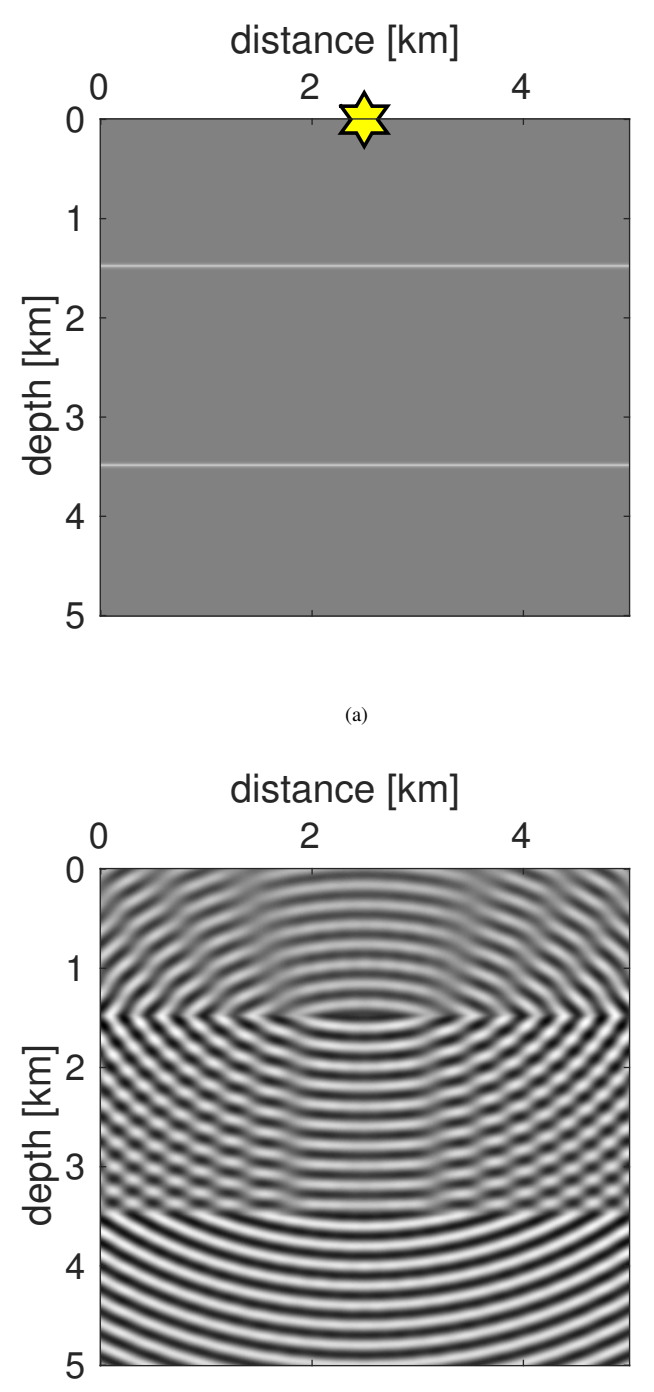

(b)

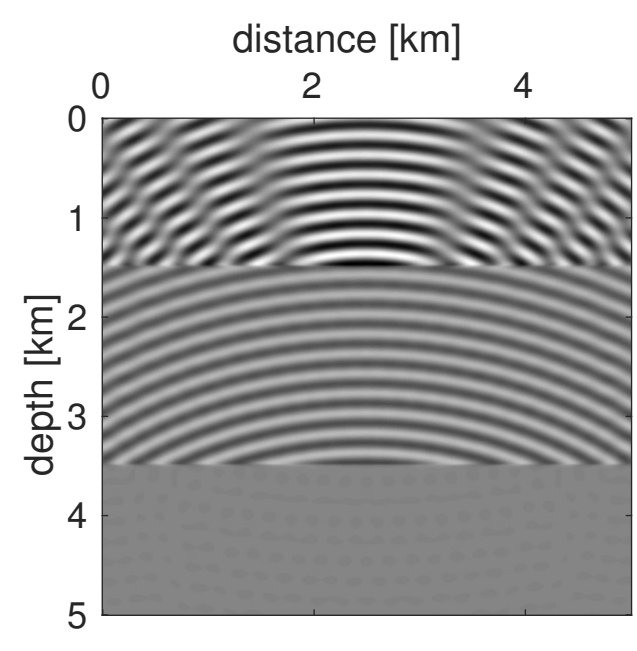

(c)

Figure 2: Application of ENBS in frequency domain using the Helmholtz solver: (a) The velocity perturbation, where the yellow star represents the source position. The real part of the scattered wavefield using (b) the conventional Born modeling, @2Q11sinsE(e) ENBS.

SEG International Exposition and 87th Annual Meeting

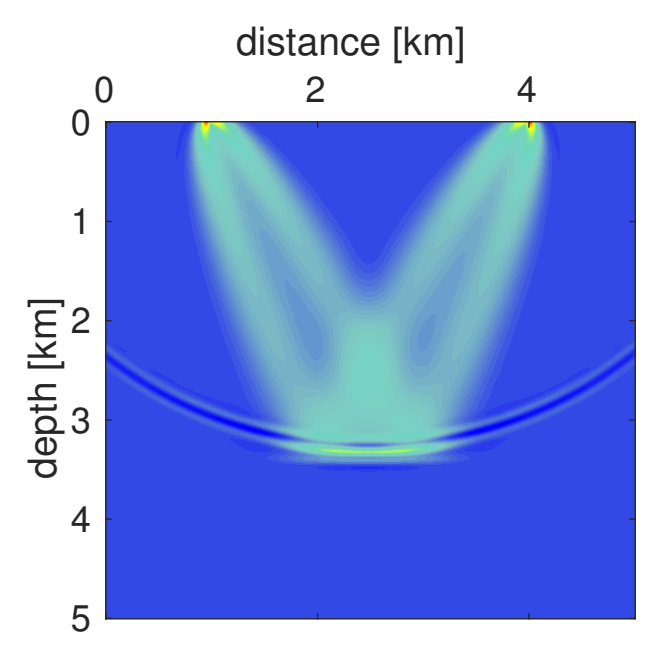

(a)

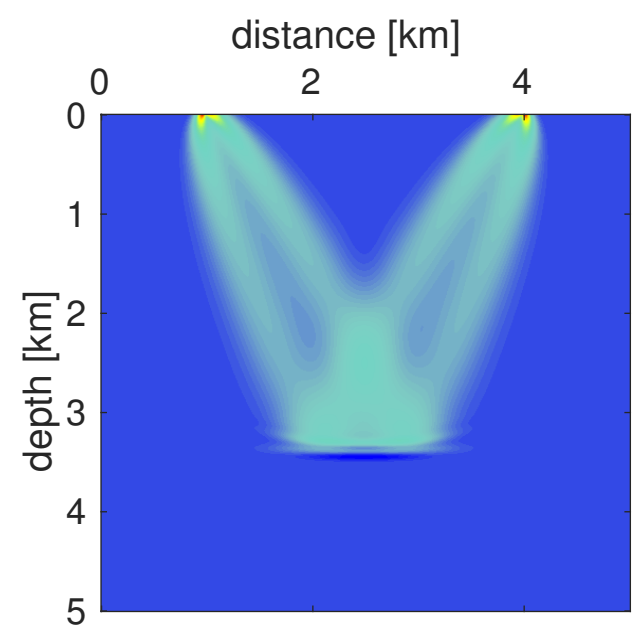

(b)

Figure 3: Application of ENBS in reflection waveform inversion: wavepath-based gradients computed using (a) the conventional born modeling, and using (b) ENBS. 


\section{EDITED REFERENCES}

Note: This reference list is a copyedited version of the reference list submitted by the author. Reference lists for the 2017 SEG Technical Program Expanded Abstracts have been copyedited so that references provided with the online metadata for each paper will achieve a high degree of linking to cited sources that appear on the Web.

\section{REFERENCES}

Irabor, K., and M. Warner, 2016, Reflection FWI: 81st Annual International Meeting, SEG, Expanded Abstracts, 1136-1140, https://doi.org/10.1190/segam2016-13944219.1.

Liu, F., G. Zhang, S. A. Morton, and J. P. Leveille, 2011, An effective imaging condition for reverse-time migration using wavefield decomposition: Geophysics, 76, S29-S39, https://doi.org/10.1190/1.3533914.

Rocha, D., N. Tanushev, and P. Sava, 2016, Acoustic wavefield imaging using the energy norm: Geophysics, 81, no. 4, S151-S163, https://doi.org/10.1190/geo2015-0486.1.

Virieux, J., and S. Operto, 2009, An overview of full-waveform inversion in exploration geophysics: Geophysics, 74, no. 6, WCC1-WCC26, https://doi.org/10.1190/1.3238367. 